\title{
Análise do índice de cobertura da mamografia em mulheres entre 50 e 69 anos, por nível de ensino, segundo unidade de federação
}

\section{Analysis of index mammography coverage in women between 50 and 69 years by level of education, according federation unit}

Jéssica de Andrade Freitas ${ }^{1}$. Beth Gleyber Pessoa de Oliveira ${ }^{1}$. Heleson Herly Ferreira ${ }^{2}$. Sarah Rodrigues do Espírito Santo ${ }^{3}$. Rafael Henrique dos Santos ${ }^{1}$.

1 Graduando do $5^{\circ}$ semestre de Medicina da Universidade Federal do Ceará (UFC), Fortaleza, Ceará, Brasil. 2 Acadêmico do $4^{\circ}$ semestre de Medicina da Universidade Federal de Minas Gerais (UFMG). 3 Acadêmica do $4^{\circ}$ semestre de Medicina da Universidade Federal do Ceará (UFC).

\section{RESUMO}

Introdução: $O$ câncer de mama é o mais incidente na população feminina brasileira e mundial, excetuando-se o câncer de pele não melanoma, sendo também o que apresenta a maior mortalidade feminina. Objetivo: Analisar a cobertura da mamografia segundo Unidade de Federação, em mulheres entre 50 e 69 anos, por nível de ensino. Metodologia: Esse estudo transversal analisou dados do Departamento de Informática do Sistema Único de Saúde (DATASUS), sobre a Pesquisa Nacional em Saúde realizada pelo Instituto Brasileiro de Geografia e Estatística (IBGE), no ano de 2013. Foram agrupadas as mulheres de 50 a 69 anos que realizaram o exame de mamografia a menos de dois anos, e subdivididas em quatro graus de escolaridade. Resultados: Verificouse que os índices de cobertura do exame no Brasil $(60,00 \%)$ e em cada Estado da Federação, tais como, Maranhão (31,90\%), Pará $(34,80 \%)$, Ceará $(41,10 \%)$ e Distrito Federal (68,70\%), encontram-se abaixo das recomendações da Organização Mundial de Saúde. Além disso, a cobertura aumenta à medida que cresce o grau de instrução dessas mulheres. Conclusão: Há uma distribuição heterogênea da cobertura de mamografia no Brasil e no Ceará, que se relaciona a diversos aspectos sociais, notadamente ao nível de escolaridade das mulheres.

Palavras-chave: Mamografia. Diagnóstico precoce. Neoplasias da mama. Cobertura de serviços de saúde.

\section{ABSTRACT}

Introduction: Breast cancer is the most frequent in the Brazilian's women worldwide, except for non-melanoma skin cancer, also being, which has the highest female mortality. Objective: To analyze the coverage of mammography according Federation Unit in women with age between 50 to 69, by level of education. Methods: This transversal study analyzed data from the Department of the Unified Health System (DATASUS) on the National Health Research conducted by the Brazilian Institute of Geography and Statistics (IBGE), in 2013. Were grouped women with age between 50 and 69 years that did mammography examination in the last years,and divided in four levels according of education. Results: If was found that the examination of the coverage rates in Brazil (60.00\%) and in every state of the Federation, such as Maranhão (31.90\%), Pará (34.80\%), Ceará (41.10 \%) and Federal District (68.70\%), are down those of the World Health Organization recommendations. Moreover, coverage increases as the level of instruction increases these women. Conclusion: There is a heterogeneous distribution of mammography coverage in Brazil and in Ceará, relates to various social aspects, notably the educational level of the women.

Keywords: Mammography. Early diagnosis. Breast neoplasms. Health services coverage.

Autor correspondente: Jéssica de Andrade Freitas, 1016, apartamento 206, Condomínio Lígia Cavalcante, Rodolfo Teófilo, Fortaleza, Ceará. CEP: 60430-372.Telefone: +55 85 98171-8441/99777-7619. E-mail: jessicaandradefreitas@hotmail.com

Conflito de interesses: Não há qualquer conflito de interesses por parte de qualquer um dos autores.

Recebido em: 15 Nov 2015; Revisado em: 29 Abr 2016; Aceito em: 23 Mai 2016. 


\section{INTRODUÇÃO}

Com exceção do câncer de pele não melanoma, o câncer de mama é o mais incidente em mulheres, representando $25 \%$ do total de casos de câncer no mundo em 2012 (1,7 milhão de casos). Consolida-se como a quinta causa de morte por câncer em geral (522.000 óbitos), o nono tipo de morte mais frequente no mundo e a maior causa de óbitos em mulheres entre todas as neoplasias malignas. ${ }^{1}$

No Brasil, também é o câncer mais incidente em mulheres, exceto na região Norte, onde o câncer do colo uterino ocupa a primeira posição, com taxa de incidência de 23,6 casos por 100.000 habitantes. Dados do Instituto Nacional do Câncer (INCA) estimam que em 2014 foram diagnosticados 57.120 casos novos (20,8\% dos diagnósticos de câncer, com exceção de pele não melanoma), o que representa a taxa de incidência de 56,1 casos por 100.000 mulheres. Foram registrados pelo Sistema de Informação de Mortalidade (SIM) 14.388 óbitos, sendo 181 homens e 14.207 mulheres, uma taxa de mortalidade de 13,5 por 100.000 mulheres. $^{2}$

Cabe ressaltar que, os valores entre as taxas de incidência e mortalidade no Brasil são maiores que nos países desenvolvidos, o que sugere a maior cobertura do rastreamento, diagnóstico precoce e tratamento da doença, nos países ditos de primeiro mundo. ${ }^{3}$

Atualmente, entre os métodos disponíveis para rastreamento do câncer de mama, o mais indicado é a mamografia, pois é um exame garantido pelo SUS, com alta sensibilidade, eficácia e simplicidade. $^{4}$

Ao analisar a literatura, é possível verificar que não existe concordância entre a faixa etária indicada para a realização da mamografia. ${ }^{5}$ O Instituto Nacional do Câncer (INCA) recomenda o exame às mulheres de 50 a 69 anos a cada dois anos. ${ }^{6}$ A Sociedade Brasileira de Mastologia, por sua vez, recomenda a realização da mamografia de rotina anualmente para mulheres a partir dos 40 anos de idade. ${ }^{7}$ A lei $n^{0} 11.664$, de 29 de Abril de 2008, regulamenta a realização de exame mamográfico a todas as mulheres a partir dos 40 anos de idade pelo Sistema Único de Saúde (SUS). ${ }^{8}$ O rastreamento do câncer de mama no Brasil não é populacional, somente se realiza de maneira a caracterizar um rastreamento oportunístico. ${ }^{5}$

É notável que, apesar do aumento no número de mulheres que fizeram a mamografia, de acordo com a Pesquisa Nacional em Saúde (PNS) de 2013, os índices ainda não atingiram o recomendado pela Organização Mundial de Saúde (OMS), que é de $70 \%$ da população na faixa etária entre 50 e 69 anos. Além disso, foi observado que nos estados do Norte e do Nordeste há um número menor de mulheres que realizam a mamografia, quando comparado com os da região Sul e no Sudeste. Este aspecto reflete, em parte, a desigualdade brasileira em relação ao índice de desenvolvimento humano e outros indicadores sociais que mostram a grande diferença de realidades entre as macrorregiões do Brasil. ${ }^{9}$

A mamografia é uma importante ferramenta para o diagnóstico precoce do câncer de mama e a análise da sua cobertura, obtida por pesquisas domiciliares, serve de base para futuras intervenções. As desigualdades sociais no acesso e uso de serviços de saúde no Brasil manifestam-se nos diferentes índices de cobertura observados nas pesquisas nacionais do Instituto Brasileiro de Geografia e Estatística (IBGE). ${ }^{10}$

Dessa forma, o objetivo desse trabalho é analisar o índice de cobertura do exame de mamografia em mulheres entre 50 e 69 anos, que relataram ter realizado mamografia há menos de dois anos, por níveis de escolaridade, em unidades de federação do Brasil.

\section{METODOLOGIA}

Trata-se de um estudo epidemiológico ecológico com base no acesso e na análise dos dados obtidos pelo Departamento de Informática do Sistema Único de Saúde (DATASUS).

A busca se concentrou na Pesquisa Nacional em Saúde (PNS) realizada pelo Instituto Brasileiro de Geografia e Estatística (IBGE) em 2013, referente ao módulo de cobertura da mamografia entre mulheres de 50 e 69 anos, que relataram ter realizado mamografia há menos de dois anos, separado por nível de instrução escolar.

As mulheres foram agrupadas, de acordo com ensino, em quatro níveis:

- Grau 1: sem instrução e fundamental incompleto;

- Grau 2: fundamental completo e médio incompleto;

- Grau 3: médio completo e superior incompleto;

- Grau 4: superior completo.

\section{Levantamento dos dados da PNS}

A pesquisa da população é feita através de entrevista nos domicílios, exceto com moradores de quartéis, bases militares, alojamentos, acampamentos, embarcações, penitenciárias, colônias penais, presídios, cadeias, asilos, orfanatos, conventos e hospitais. A amostra da Pesquisa Nacional de Saúde (PNS) é uma subamostra da Amostra Mestra do Sistema Integrado de Pesquisas Domiciliares (SIPD) do IBGE. ${ }^{11}$

\section{Área de estudo}

De acordo com dados do IBGE, o Brasil ocupa uma área de 8.515.767,049 km², com 5.570 municípios e população de mais de 205.030.210 habitantes.

O estado do Ceará, situado na Região Nordeste do país, com capital Fortaleza, apresenta área de $148.886,308 \mathrm{~km}^{2}$, com 184 municípios e população estimada para 2015 de 8.904.459 habitantes.

\section{RESULTADOS}

Os estudos dos dados da PNS de 2013 permitiram a observação da cobertura da mamografia nas unidades de federação do 
Brasil. A partir disso, pode-se destacar que a cobertura do exame, sem levar em consideração os níveis de instrução das mulheres, foi menor nos Estados do Maranhão (31,90\%) e Pará (34,80\%).

Os melhores resultados foram verificados no Distrito Federal $(68,70 \%)$ e no Estado de São Paulo (73,90\%), enquanto a média do Brasil se manteve com $(60,00 \%)$ de cobertura do exame de mamografia (Gráfico 1).

Gráfico 1. Cobertura do exame de mamografia sem considerar níveis de escolaridade das mulheres, nos Estados de menores (Maranhão e Pará) e maiores (Distrito Federal e São Paulo) índices de cobertura, no Ceará e no Brasil.

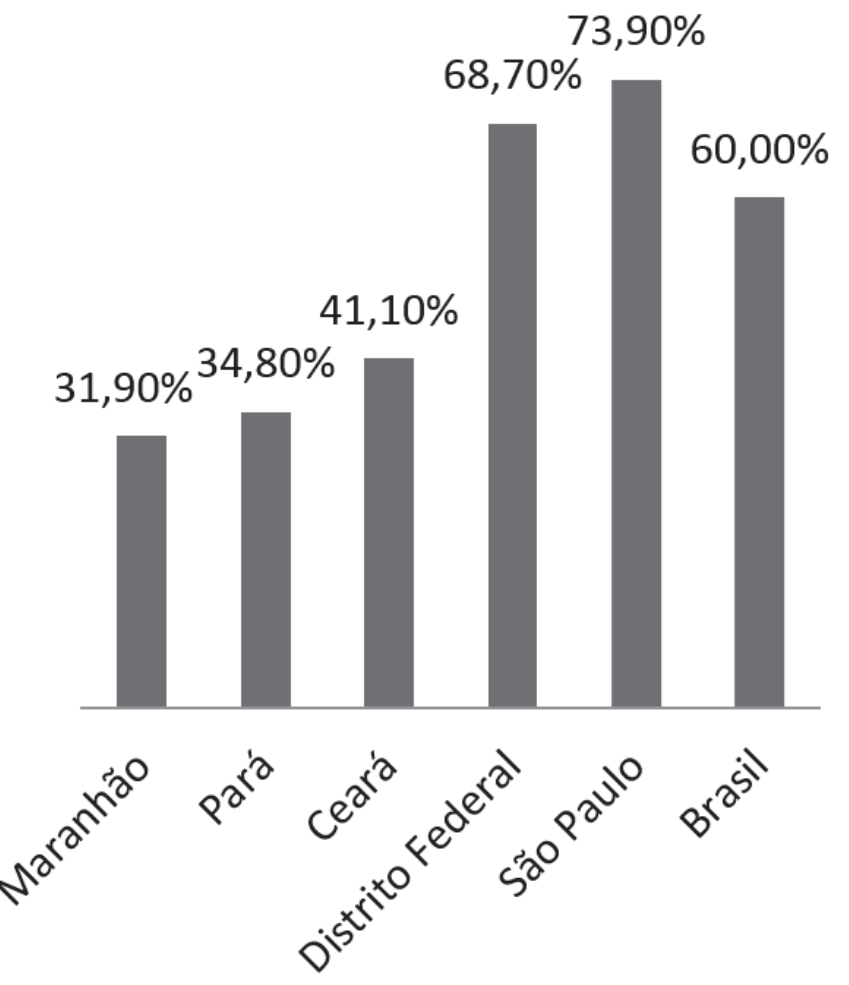

Fonte: elaborado pelos autores.

Nesse contexto, o Ceará encontra-se na $20^{\circ}$ posição $(41,1 \%)$ no ranking em ordem decrescente de cobertura do exame, dentre os 26 estados da federação e o Distrito Federal. Ao considerar os graus de escolaridades das mulheres que participaram da pesquisa, observa-se $32,5 \%$ de cobertura da mamografia no Ceará e 50,9\% no Brasil para mulheres sem instrução e fundamental incompleto (Grau 1); para fundamental completo e médio incompleto (Grau 2) o Ceará apresentou-se sem dados e o Brasil com 60,6\%; a cobertura para médio completo e superior incompleto (Grau 3) foi no Ceará e no Brasil, respectivamente, $56,9 \%$ e $72,4 \%$. Por último, para superior completo (Grau 4) a cobertura foi de $73,1 \%$ no Ceará e $80,9 \%$ no Brasil (Tabela 1).
Tabela 1. Porcentagem de cobertura do exame de mamografia por níveis de escolaridade.

\begin{tabular}{lllll}
\hline & Maranhão & Ceará & São Paulo & Brasil \\
\hline Grau 1 & $23,3 \%$ & $32,5 \%$ & $68 \%$ & $50,9 \%$ \\
Grau 2 & & & $68,1 \%$ & $60,6 \%$ \\
Grau 3 & $71,5 \%$ & $56,9 \%$ & $81,8 \%$ & $72,4 \%$ \\
Grau 4 & $63,9 \%$ & $73,1 \%$ & $83,8 \%$ & $80,9 \%$ \\
\hline
\end{tabular}

Nota: Grau 1: sem instrução e fundamental incompleto; Grau 2: fundamental completo e médio incompleto; Grau 3: médio completo e superior incompleto; Grau 4: superior completo. Espaços em branco não apresentaram dados específicos para o grau

Fonte: elaborada pelos autores.

\section{DISCUSSÃO}

De acordo com os resultados, pode-se verificar que os índices de cobertura do exame de mamografia na maior parte dos casos estão abaixo do indicado pela Organização Mundial de Saúde (OMS) que preconiza a realização do exame a 70\% das mulheres entre 50 e 69 anos. $^{9}$

Pode-se destacar a grande variação na percentagem de cobertura do exame de mamografia entre os 26 estados da federação e o Distrito Federal, por exemplo, a menor cobertura sendo 31,90\% no Maranhão e a maior 73,90\% em São Paulo.

Nesse contexto a média brasileira de $60,00 \%$, além de não corresponder homogeneamente aos Estados do país, se faz abaixo das recomendações previstas pela OMS. Além dessa discrepância entre Estados pode-se destacar que apenas São Paulo, sem levar em consideração os graus de escolaridade das mulheres que realizam o exame de mamografia, conseguiu se enquadrar nas normas da OMS.

Ao analisar os níveis de escolaridade das mulheres do Brasil, se torna praticamente unânime o crescimento da cobertura da realização do exame à medida que crescem os graus de instrução dessas mulheres.

Nesse contexto, o Ceará se encontra entre os sete Estados com piores números de abrangência da mamografia. No Estado, é notável a diferença entre Grau $1(32,5 \%)$ e Grau 4 (73,1\%). Diferente do que se verifica em Estados da Região Sul e Sudeste, como por exemplo, em São Paulo (50,9\% - 80,9\%), onde os valores têm uma menor variação.

\section{CONCLUSÃO}

É possível inferir que os índices de cobertura do exame de mamografia no Brasil estão abaixo dos números esperados e por se tratar do câncer que mais mata mulheres no mundo, o rastreamento e diagnóstico precoce do câncer de mama pela mamografia devem ser priorizados pelas políticas promotoras de saúde.

Por conseguinte, são necessárias medidas para reduzir a 
heterogeneidade dos resultados obtidos em cada Estado do país e também em relação aos diferentes níveis de escolaridade verificados, com diminuição das desigualdades sociais, distribuição igualitária de mamógrafos e conscientização da população em relação aos benefícios ao realizar a mamografia.

\section{REFERÊNCIAS}

1. International Agency for Research on Cancer. Globocam 2012: estimed cancer incidence, mortality and prevalence worldwide in 2012 [Internet]. Lyon: World Health Organization; c2016. [acesso 2015 nov 21 nov]. Disponível em: http://globocan.iarc.fr/Pages/ fact_sheets_cancer.aspx

2. Facina T. Estimativa 2014: incidência de Câncer no Brasil. Revista Brasileira de Cancerologia [Internet]. 2014 [acesso 2015 nov 21];60(1):63-4. Disponível em: http://www.inca.gov.br/rbc/n_60/ v01/pdf/11-resenha-estimativa-2014-incidencia-de-cancer-no-brasil. pdf

3. Instituto Nacional de Câncer José Alencar Gomes da Silva (INCA). Controle do Câncer de Mama: conceito e magnitude [Internet]. Rio de Janeiro: INCA; c2016 [acesso 2015 nov 21]. Disponível em: http:// www2.inca.gov.br/wps/wcm/connect/acoes_programas/site/home/ nobrasil/programa_controle_cancer_mama/conceito_magnitude

4. Perry N, Broeders M, Wolf C, Törnberg S, Holland R, von Karsa L. European guidelines for quality assurance in breast cancer screening and diagnosis. 4th ed. Luxembourg: European Commission; 2006. $432 \mathrm{p}$.

5. Urban LA, Schaefer MB, Dakir L, Duarte DL, Santos RP, Maranhão $\mathrm{NM}$, et al. Recomendações do Colégio Brasileiro de Radiologia e Diagnóstico por Imagem, da Sociedade Brasileira de Mastologia e da Federação Brasileira das Associações de Ginecologia e Obstetrícia para rastreamento do câncer de mama por métodos de imagem. Radiol Bras. 2012;45(6):334-9.

6. Instituto Nacional de Câncer José Alencar Gomes da Silva (INCA). Controle do Câncer de Mama: detecção precoce [Internet]. Rio de Janeiro: INCA; c2016 [acesso 2015 nov 21]. Disponível em: http://
Por fim, atitudes que visem aumentar a cobertura do exame acabam por diminuir a taxa de mortalidade da neoplasia de mama, aumentar a sobrevida e as chances de cura. Tudo isso devido ao diagnóstico precoce que deixa de ser oportunístico e transforma-se em algo organizado, capaz de beneficiar toda a população feminina.

www2.inca.gov.br/wps/wcm/connect/acoes_programas/site/home/ nobrasil/programa_controle_cancer_mama/deteccao_precoce

7. Damin AP. Rastreamento do câncer de mama: quais as tendências atuais? Rev HCPA. 2012;32(2):129-130.

8. Brasil. Lei $n^{\circ}$. 11.664, de 29 de abril de 2008. Dispõe sobre a efetivação de ações de saúde que assegurem a prevenção, a deteç̧ão, o tratamento e o seguimento dos cânceres do colo uterino e de mama, no âmbito do Sistema Único de Saúde - SUS. Brasília: Casa Civil; 2008. [acesso 2015 nov 21]. Disponível em: http://www.planalto. gov.br/ccivil_03/_ato2007-2010/2008/lei/111664.htm

9. Nota Oficial. Sala de Imprensa. Sociedade Brasileira de Mastologia, 2014[acessado 2015 nov 21, 18:21]. Disponível em http://www.sbmastologia.com.br/index.php?option=com content\&view=article\&id=754:nota-oficial-sociedade-brasileira-demastologia\&catid $=168 \&$ Itemid $=887$

10. Viacava,F, Souza PR Jr, Moreira RS. Estimativas da cobertura de mamografia segundo inquéritos de saúde no Brasil. Rev Saúde Pública. 2009;43 Supl 2:117-25.

11. Instituto Brasileiro de Geografia e Estatística (IBGE). Pesquisa Nacional em Saúde 2013: percepção do estado de saúde, estilos de vida e doenças crônicas - Brasil, grandes regiões e unidades de federação [Internet]. Rio de Janeiro: IBGE; 2014 [acesso 2015 nov 21]. 181 p. Disponível em: ftp://ftp.ibge.gov.br/PNS/2013/pns2013. pdf

\section{Como citar:}

Freitas JA, Oliveira BG, Ferreira HH, Espírito Santo SR, Santos RH. Análise do índice de cobertura da mamografia em mulheres entre 50 e 69 anos, por nível de ensino, segundo unidade de federação. Rev Med UFC. 2016 jan-jun;56(1):14-17. 